\title{
Enhancing the rate of tidal disruptions of stars by a self-gravitating disc around a massive central black hole
}

\author{
V. Karas ${ }^{1, a}$ and L. Šubr ${ }^{2}$ \\ ${ }^{1}$ Astronomical Institute, Academy of Sciences of Czech Republic, Prague, Czech Republic \\ ${ }^{2}$ Astronomical Institute, Faculty of Mathematics and Physics, Charles University, Prague, Czech Republic
}

\begin{abstract}
We further study the idea that a self-gravitating accretion disc around a supermassive black hole can increase the rate of gradual orbital decay of stellar trajectories (and hence tidal disruption events) by setting some stars on eccentric trajectories. Cooperation between the gravitational field of the disc and the dissipative environment can provide a mechanism explaining the origin of stars that become bound tightly to the central black hole. We examine this process as a function of the black hole mass and conclude that it is most efficient for intermediate central masses of the order of $\sim 10^{4} M_{\odot}$. Members of the cluster experience the stage of orbital decay via collisions with an accretion disc and by other dissipative processes, such as tidal effects, dynamical friction and the emission of gravitational waves. Our attention is concentrated on the region of gravitational dominance of the central body. Mutual interaction between stars and the surrounding environment establishes a non-spherical shape and anisotropy of the nuclear cluster. In some cases, the stellar sub-system acquires ring-type geometry. Stars of the nuclear cluster undergo a tidal disruption event as they plunge below the tidal radius of the supermassive black hole.
\end{abstract}

\section{INTRODUCTION}

Tidal disruptions of stars near a supermassive black hole (SMBH) occur during close encounters, i.e., when a satellite star on an eccentric trajectory plunges below the critical (tidal) radius $R_{\mathrm{t}} \sim 10^{11}\left(M / M_{*}\right)^{1 / 3}\left(R_{*} / R_{\odot}\right) \mathrm{cm}$, where $M_{*}$ and $R_{*}$ denote the mass and the radius of the satellite $[2,13,15]$. In this context it is of a particular interest to study processes that can influence the orbital motion of stars in the close vicinity of the central black hole. Here we will summarize our prior works $[5,7,16,17]$ on various perturbation effects that can cause the orbital decay, so that stars of the nuclear cluster migrate towards the centre. Near the center the stars eventually plunge below the horizon, or they become disrupted by tidal forces of the supermassive black hole.

We can imagine a nuclear cluster around a galactic core as a system consisting of a central black hole, an accretion disc and a dense stellar cluster. These three components are known to exist in most of galactic nuclei, in particular active galactic nuclei where the accretion flow provides fuel for the core activity. The evidence is more complicated in the case of our Galactic Center (despite its relative proximity) because there is no standard accretion disc present [3]. However, we can still clearly resolve the inflowing material and numerous effects of the past activity $[10,18]$, which also prove the relevance of the threecomponent scenario. The three mentioned subsystems are in mutual interaction with each other, and the role of this interaction is essential for their long-term behaviour. Figure 1 shows a schematic view of the model under consideration.

A number of approximations need to be imposed in order to keep our model simple and tractable. The aim is to examine the structure of a stellar system in the region

\footnotetext{
a e-mail: vladimir.karas@cuni.cz
}

of black hole gravitational dominance $\left(R_{\mathrm{h}}\right)$, including the dissipative effects due to a gaseous disc.

Two regions of the cluster can be distinguished according to the characteristic time-scale of processes dominating the stellar motion. The outer cluster is thought to reach a gravitationally relaxed state [1] that forms a reservoir of fresh satellites which are continuously injected inwards. On the other hand, the inner cluster is defined as a region where direct physical (hydrodynamical) star-disc collisions take place.

\section{STARS NEAR A SMBH: INDIVIDUAL ORBITS AND COLLECTIVE EFFECTS}

Stars dissipate their orbital energy and momentum by successive passages through gaseous medium of an accretion disc. On an inclined trajectory each encounter with the disc slab slightly changes the orbital parameters. The overall trend is to circularize orbits and to decline them into the plane of the disc. However, as we shall demonstrate later on, this prevailing trend is superposed with periods of enhanced orbital eccentricity which is caused by the gravitational influence of the very same accretion disc. Both effects are proportional to the mass distribution in the accretion disc, and so they have to be treated simultaneously. Taking one of the effects into account and ignoring the other one would be an inconsistent approach.

The relaxation time-scale can be estimated by substituting $\sigma$ with the Keplerian velocity and setting $C \ln \Lambda \approx 10^{2}$ in $t_{\mathrm{r}}$ :

$$
t_{\mathrm{r}}=1.3 \times 10^{19}\left(\frac{M_{*}}{M_{\odot}}\right)^{-2}\left(\frac{n(r)}{10^{6} \mathrm{pc}^{-3}}\right)^{-1}\left(\frac{r}{R_{\mathrm{g}}}\right)^{-\frac{3}{2}} \mathrm{yr} .
$$

In the case of Bahcall-Wolf distribution, the stellar number density follows a power-law radial profile, $n(r) \propto r^{-7 / 4}$. 

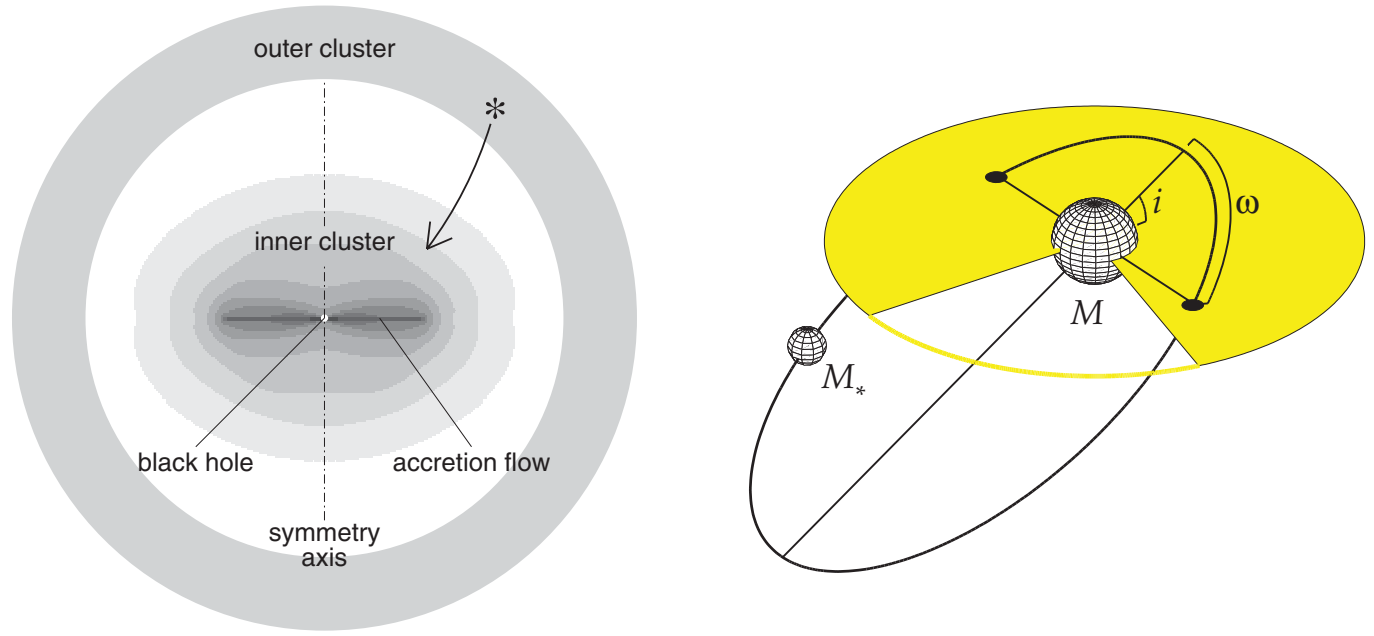

Figure 1. A schematic drawing of the model adopted in computations (left panel). Its main ingredients are a massive compact body in the centre, an accretion disc or a torus, and a stellar cluster. The latter one has been divided here into two components: an inner cluster is formed by stars on orbits bound gravitationally to the centre and evolving due to interactions with the disc. The outer cluster serves as a reservoir of satellites injected into the inner region. Gray levels used within the inner cluster indicate the number density of the satellites, as it follows from computations. Also shown is the geometrical situation of an orbit followed by an exemplary satellite in the inner cluster (in the right panel).

In the velocity space we assume that the distribution function of the boundary cluster corresponds to the Maxwell velocity dispersion.

In order to simulate the impact of hydrodynamical interaction between the satellite and the disc medium we adopt a simple approximation of instantaneous changes of the orbiter velocity components after each crossing the equatorial plane: the momentum change is proportional to the geometrical cross-section of the stellar body and its local velocity relative to the gas $[5,14]$. This approach can be adequate in the situation when the disc is geometrically thin $(H \ll R)$ and the relative velocity of satellite and the disc medium exceeds speed of sound in the surrounding environment. Under these assumptions we can work with vertically integrated quantities.

Characteristic time of aligning stellar orbits with the disc is $t_{\mathrm{d}}(a) \approx t_{0} M_{8}\left(\Sigma_{*} / \Sigma_{\odot}\right)^{-1}\left(a / R_{\mathrm{g}}\right)^{q} \mathrm{yr}$, where $a$ is semimajor axis, $t_{0}$ and $q$ are constants (order of unity for the standard disc model) and $M_{8} \equiv M_{\bullet} /\left(10^{8} M_{\odot}\right)$ is the fiducial value of the central mass [5]. Different modes of radial migration are relevant for stars in the disc plane depending whether a star succeeds to open a gap in the disc medium or if it instead remains embedded entirely and proceeds via excitation of density waves. Finally, we computed the gravitational field of the disc and took its effect also into account for the long-term orbital decay of stellar trajectories.

In order to construct a steady-state cluster we assume a continued supply of stellar bodies from the reservoir (at rate inversely proportional to the relaxation time). Our computational scheme [17] allows us to further distinguish between two subsamples of the inner cluster: the dragged inner cluster consists of stars on orbits crossing the disc periodically; the embedded inner cluster is formed by stars entirely aligned with the disc. The outer stellar cluster can be characterized by the number density $n_{0}=10^{6} \mathrm{pc}^{-3}$ and velocity dispersion $\sigma_{\mathrm{c}}=200 \mathrm{~km} / \mathrm{s}$.
In ref. [17] we studied the density structure of the cluster which is established as a result of the mutual interaction of its components (see figure 2). Majority of stars forming the embedded cluster sink to the centre in the regime of density waves, hence $v_{r} \propto r^{1 / 2}$ and $n(a) \propto a^{-1 / 2}$. In the dragged cluster, the orbital decay leads to the corresponding number density $n(a) \propto a^{5 / 4}$. The asymptotic profile $\propto a^{1 / 4}$ of the outer cluster is determined by the initial Bahcall-Wolf distribution.

The most interesting conclusion from fig. 2 concerns the formation of a highly flattened stellar subsystem in the inner region surrounding the central SMBH. In fact, it resembles a distinct feature of the highly flattened distribution of stars with high number density which was originally reported within the kiloparsec scale of the Galactic bulge $[8,11]$. In our scenario the origin of such a flattened stellar system would be associated with the hydrodynamical dissipation by the dusty/gaseous disc together with its gravitational effect. In the context of tidal disruption events, another effect of this interaction is that a subset of stars on eccentric orbits is brought close to the central black hole where they plunge below the tidal radius. It will be interesting to compare our results with a recent work [4] where a vertically extended accretion disc is considered and the star-disc ram pressure effects are taken into account in a more realistic manner.

\section{STELLAR CAPTURE RATES FROM HIGHLY ECCENTRIC ORBITS}

In ref. [7] we characterised the influence of highly eccentric orbits on the star-capture rate by introducing fractional rates, given by the number of stars that exhibit pericentre distances below a certain threshold radius $R_{\min }$. We denote this fraction $\mathcal{F}_{2}\left(R_{\min } ; a, C_{1}\right)$ and write it in the 

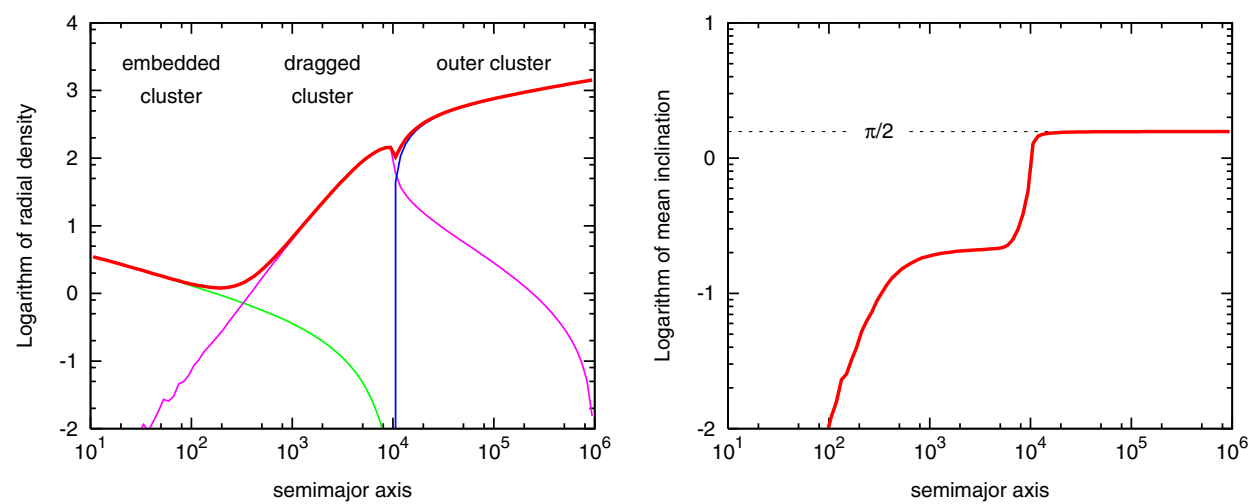

Figure 2. Structure of the nuclear cluster interacting with an embedded accretion disc. The graphs on the left side shows the distribution of semi-major axes of stars forming a dense nuclear cluster, modified by interaction with the gas-pressure dominated standard accretion disc. The broken power-law profile is established by different types of interaction governing different regions of phase space. The three stellar sub-samples are present in our model and they can be distinguished in the plot: the outer reservoir (magenta), the inner part of the cluster which is dragged by the disc (blue), and a subsample of stars fully embedded in the disc (green). Further, in the right panel, a graph of mean orbital inclination $\langle i\rangle$ is shown. The latter plot exhibits the effect of flattening of the nucler cluster. The outer reservoir is spherically symmetric $(\langle i\rangle=\pi / 2)$, the dragged cluster is somewhat flattened $(0<\langle i\rangle<\pi / 2)$, and the embedded population is located in the disc plane $(\langle i\rangle=0)$. This figure adapted from ref. [6].
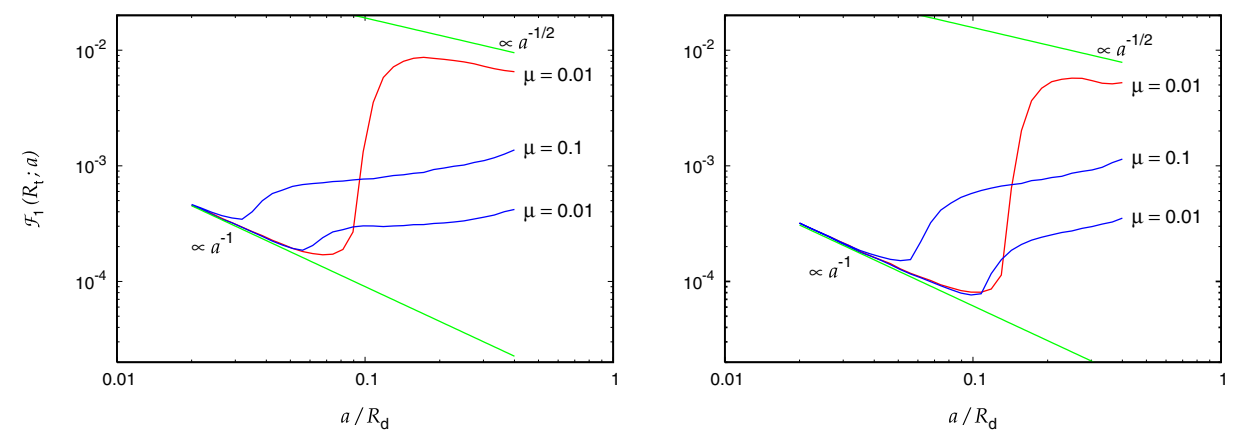

Figure 3. Fraction $\mathcal{F}_{1}\left(R_{\mathrm{t}} ; a\right)$ of stars plunging close to the black hole tidal radius. Stellar orbits are perturbed by a massive ring of radius $R_{\mathrm{d}}$ and mass $M_{\mathrm{d}}=\mu M_{\bullet}$ (indicated with curves). Red curve is for the post-Newtonian (PN1) approximation of the central mass gravity field; the effect of the outer stellar cluster is neglected. Blue curves represent two cases in which the cluster is taken into account (they differ by the disc mass parameter $\mu$ ). Two power-law lines correspond to the lower and the upper analytical estimates [7]. Vertical dotted lines indicate the terminal values of semimajor axis $a_{\mathrm{t}}$ (with $\mu=0.01$ and 0.1 , respectively). These are critical values below which Kozai's phenomenon is suppressed. The eccentricity oscillations persist for $a>a_{\mathrm{t}}$. Parameter values assumed in this plot are: in the left panel $M_{\bullet}=10^{4} M_{\odot} ;$ in the right panel $M_{\bullet}=10^{5} M_{\odot}$.

form

$\mathcal{F}_{2} \equiv \frac{1}{D_{2}} \int_{0}^{\sqrt{1-C_{1}^{2}}} \mathrm{~d} e \int_{0}^{2 \pi} \mathrm{d} \omega \Theta\left(e_{\max }-e_{\min }\right) D_{\mathrm{f}}\left(a, C_{1}, e, \omega\right)$,

where $e_{\min } \equiv 1-R_{\min } / a$ and $e_{\max }\left(a, C_{1}, e, \omega\right)$ are the minimum and the maximum eccentricity on a given trajectory, respectively. Further, we define

$$
\mathcal{F}_{1}\left(R_{\min } ; a\right) \equiv \frac{1}{D_{1}(a)} \int_{0}^{1} \mathrm{~d} C_{1} \mathcal{F}_{2}\left(R_{\min } ; a, C_{1}\right) D_{2}\left(a, C_{1}\right)
$$

and

$$
\mathcal{F}\left(R_{\min }\right) \equiv \int_{a_{\min }}^{a_{\max }} \mathrm{d} a \mathcal{F}_{1}\left(R_{\min } ; a\right) D_{1}(a)
$$

( $D_{1}$ and $D_{2}$ are normalization factors). The integral quantities $\mathcal{F}_{\rangle}$allow us to describe the corresponding fractions of the star cluster population that reach down to a small distance from SMBH. In particular we can use them to characterize the class of trajectories plunging below $R_{\mathrm{t}}$. We can use the averaging technique to find parameters of the orbits that reach high eccentricities. Then, we integrate the equations of motion directly for those highly eccentric orbits.

Kozai's resonance mechanism $[9,12]$ operates in a system where the Newtonian central potential is perturbed by a small axially symmetric term (the disc potential in our case). The effect is known to be damped by other perturbations that may influence the motion of stars, namely, it is suppressed when the central field is nonNewtonian (because of the precession of orbits). Therefore we wanted to learn whether the Kozai mechanism is still relevant for the long-term dynamics of nuclear stars, even if relativistic pericentre advance and the effect of the nuclear cluster are taken into account.

\section{FRACTION OF STARS OF THE NUCLEAR CLUSTER PLUNGING BELOW THE TIDAL RADIUS OF SMBH}

Figure 3 shows the fraction $\mathcal{F}_{1}\left(R_{\mathrm{t}} ; a\right)$ [7]. We find that the damping effects are weak if the mass of the axisymmetric 
component (i.e. the gravitational perturbation by a selfgravitating accretion disc) is high. By increasing the mass parameter $\mu$ (the ratio of the disc mass to the black hole mass) the curve of $\mathcal{F}_{1}\left(R_{\mathrm{t}} ; a\right)$ gradually gets shifted to higher values. Simultaneously, $a_{\mathrm{t}}$ is diminished. As a consequence of these dependencies, more stars reach the tidal radius for lower masses of the central body than for higher masses.

As the mass of the SMBH decreases the interplay of the Kozai mechanism and the two-body relaxation becomes more complicated. However, we still expect that the character of the angular momentum changes tends to enhance the rate of tidal disruptions. We imagine that consumption of stars by the central intermediate-mass black hole (IMBH) is a transient process that enhances their activity during those periods when stars are supplied into the loss cone and then efficiently brought onto eccentric orbits. Permanent replenishment of this enlarged loss cone is not critical for the process considered here.

In the case of IMBH the resonance mechanism complements the role of stellar encounters in the process of feeding the black hole. If the total mass of the cluster is about $M_{\bullet} \simeq 10^{4} M_{\odot}$, we expect the enhancement of the disruption rate $\simeq M_{\odot}$ per $10^{3}$ yrs (with $\mu=0.1$ ). Disrupted stars then provide material for accretion, thereby triggering a transient luminous phase of the object.

\section{CONCLUSIONS}

Active galactic nuclei are fed by accretion process onto a super-massive black hole. An accretion disc is an essential part of the model. However, the role of stars is also crucial and cannot be neglected. Orbiting and gradually sinking stars are mutually interconnected with a diluted interstellar environment in the form of gas and dust, and the two components influence each other. As a result of the interaction, the rate of stars sinking to the black hole is enhanced and the number of tidal disruption events is enlarged.

Stars on highly elongated orbits are susceptible to tidal disruption and hence provide a natural source of material to replenish the inner disc. The phenomenon of star-disc interaction operates in the system even if it is disturbed by the relativistic pericentre precession and by the gravity of the nuclear cluster. Therefore we can conclude that the presence of a gaseous disc of a small but non-zero mass, $0<M_{\mathrm{d}}<M_{\bullet}$, helps to drag stars to the black hole, thereby feeding the centre and simultaneously providing material that sustains and replenishes the disc itself. Disruption of stellar bodies and subsequent accretion of the remnant gas are among likely mechanisms feeding supermassive black holes that are embedded in a dense nuclear star cluster.

The stellar motion is obviously less sensitive to nongravitational forces than the gas swirling in an accretion flow, nonetheless, the mutual interaction between stars and the interstellar environment still needs to be determined with sufficient accuracy. Assuming that hydrodynamical drag in the disc is the main perturbing agent, we showed how an initially spherical system attains a flattened configuration and how a part of the system forms a ring, typically on $\sim$ parsec scales. An enhanced rate of star-disc collisions and mutual stellar encounters near the black hole may contribute to the variability that many galactic nuclei exhibit and it may partly explain the origin of material swept out of the accretion disc.

Finally, the accretion disc driven evolution of the cluster results in a gradual modification of the stellar mass function. The effect of the satellites orbital decay changes its initial (Salpeter type) form and contributes to the separation of satellites according to their physical properties. In consequence the star cluster experiences gradual segregation of its members because stars of different size and mass sink at different pace towards the center.

Support from European COST Action MP0905 "Black Holes in a Violent Universe" (ref. 208092) and the Czech Academy of Sciences (RVO:67985815) is gratefully acknowledged.

\section{References}

[1] Bahcall J. N., Wolf R. A., 1976, ApJ 209, 214

[2] Evans C. R., Kochanek C. S., 1989, ApJ 346, L13

[3] Haas J., Šubr L., Kroupa P., 2011, MNRAS 412, 1905

[4] Just A., Yurin D., Makukov M., Berczik P. et al., 2012, ApJ, in press (arXiv:1208.4954)

[5] Karas V., Šubr L., 2001, A\&A 376, 686

[6] Karas V., Šubr L., 2004, in Proc. of the 22nd Texas Symposium on Relativistic Astrophysics (Stanford University, Stanford), p. 2103

[7] Karas V., Šubr L., 2007, A\&A 470, 11

[8] Karas V., Šubr L., Šlechta M., 2002, in Gravitation, Following the Prague Inspiration, eds. Semerák O., Podolský J., \& Žofka M. (World Scientific Publ., Singapore), p. 85

[9] Kozai Y., 1962, AJ 67, 591

[10] Kunneriath D., Eckart A., Vogel S. N., Teuben P. et al., 2012, A\&A 538, A127

[11] Launhardt R., Zylka R., Mezger P. G., 2002, A\&A 384, 112

[12] Lidov M. L., 1962, Planet. Space Sci. 9, 719

[13] Luminet J.-P., Marck J.-A., 1985, MNRAS 212, 57

[14] Psaltis D., 2012, ApJ, submitted (arXiv:1112.0026)

[15] Rees M. J., 1988, Nature 333, 523

[16] Šubr L., Karas V., 2005, A\&A 433, 405

[17] Šubr L., Karas V., Huré J.-M., 2004, MNRAS 354, 1177

[18] Terrier R., Ponti G., Bélanger G., Decourchelle A. et al., 2010, ApJ 719, 143 